\title{
INTEGRAL REPRESENTATIONS OF GENERALIZED LAURICELLA HYPERGEOMETRIC FUNCTIONS
}

\author{
VU KIM TUAN \\ Institute of Mathematics \\ P.O. Box 631, Вठ H \\ Hanoi, Vietnam
}

AND

AND

\author{
R.G. BUSCHMAN \\ Department of Mathematics \\ Box 3036, University Station \\ Laramie, WY 82071, USA
}

(Received August 10, 1990 and in revised form March 24, 1992)

ABSTRACT. The generalized hypergeometric function was introduced by Srivastava and Daoust. In the present paper a new integral representation is derived. Similarly new integral representations of Lauricella and Appell function are obtained.

KEY WORDS. Lauricella, Appell, integral representations, Mellin transformation.

1991 AMS SUBJECT CLASSIFICATION CODES. 33A35, 44A30

\section{INTRODUCTION.}

The generalized Lauricella function of several complex variables was introduced by Srivastava and Daoust $[7,8]$. The only integral representation which seems to be known for this function is in terms of a Mellin-Barnes integral $[6,8]$. From a fundamental result about Mellin transforms in $n$-dimensions, we obtain a new representation for the generalized Lauricella function under suitable restrictions on the parameters. In a similar manner new integral representations are obtained for the Lauricella functions $F_{A}^{(n)}, F_{C}^{(n)}$ and $F_{D}^{(n)}$, and consequently for the Appell functions $F_{1}, F_{2}$, and $F_{4}$. From these derivations it is clear that the method does not provide representations for $F_{B}^{(n)}$ and $F_{3}$.

\section{THE FUNDAMENTAL THEOREM.}

Although the following theorem is quite simple, nevertheless it has basic importance.

THEOREM. Let $f(x) x^{s-1} \in L(0, \infty)$ and

$$
M_{1}[f(x)](s)=\int_{0}^{\infty} f(x) x^{s-1} d x=f^{*}(s) .
$$

If $\operatorname{Res}_{j}>0, j=1,2, \cdots, n$ and $\operatorname{Re}\left(\sum_{j=1}^{n} s,\right)=$ Res, then

$$
\begin{gathered}
M_{n}\left[f\left(\max \left\{x_{1}, \cdots, x_{n}\right\}\right)\right]\left(s_{1}, \cdots, s_{n}\right)=\int_{0}^{\infty} \cdots \int_{0}^{\infty} f\left(\max \left\{x_{1}, \cdots, x_{n}\right\}\right) \prod_{i=1}^{n} x_{i}^{2,-1} d x_{1} \cdots d x_{n}= \\
=\frac{s_{1}+\cdots+s_{n}}{s_{1} \cdots s_{n}} f^{*}\left(s_{1}+\cdots+s_{n}\right) .
\end{gathered}
$$

Here $M_{n}$ represents the $n$-dimensional Mellin integral transformation [2].

PROOF. In fact we have

$$
\begin{aligned}
\int_{0}^{\infty} & \cdots \int_{0}^{\infty} f\left(\max \left\{x_{1}, \cdot s, x_{n}\right\}\right) \prod_{i=1}^{n} x_{i}^{s,-1} d x_{1} \cdots d x_{n}= \\
= & \sum_{i=1}^{n} \int_{0}^{\infty} f\left(x_{i}\right) \int_{0}^{x_{1}}(n-1) \int_{0}^{x_{i}} \prod_{j=1}^{n} x_{j}^{s,-1} d x_{1} \cdots d x_{n}=
\end{aligned}
$$




$$
=\sum_{i=1}^{n} \frac{s_{i}}{s_{1} s_{2} \cdots s_{n}} \int_{0}^{\infty} f\left(x_{i}\right) x_{i}^{s_{1}}++s_{n}-1 d x_{i}=\frac{s_{1}+\cdots+s_{n}}{s_{1} \cdots s_{n}} f^{*}\left(s_{1}+\cdots+s_{n}\right) .
$$

Thus the formula (2) is proved.

The only related results which we have found in the literature are those for the two-dimensional Laplace transformation in the tables of Voelker and Doetsch $[9 ;$ p. 165, $(30),(32)]$ and in the work of Černov $[4 ;$ p. 145]. In an analogous manner to our theorem, those results easily can be derived and extended to higher dimensions.

\section{THE INTEGRAL REPRESENTATION OF THE GENERALIZED LAURICELLA FUNCTION.}

The generalized hypergeometric function of Srivastava and Daoust [6] is defined by

$$
\begin{aligned}
& F_{q \cdot q_{1},}^{p p_{1}, p_{n}}\left(\begin{array}{l}
a_{p} ; c_{p_{1}}^{1} ; \cdots ; c_{p_{n}}^{n} ; x_{1}, \cdots, x_{n} \\
b_{q} ; d_{q_{1}}^{1} ; \cdots ; d_{q_{n}}^{n} ;
\end{array}\right)= \\
& =\sum_{m_{1}, m_{n}=0}^{\infty} \frac{\prod_{j=1}^{p}\left(a_{j}\right)_{m_{1}+\cdot+m_{n}}}{\prod_{j=1}^{q}\left(b_{j}\right)_{m_{1}}++m_{n}} \prod_{i=1}^{n}\left\{\frac{\prod_{j=1}^{p_{1}}\left(c_{j}^{i}\right)_{m_{1}} x_{i}^{m_{1}}}{\prod_{j=1}^{q_{1}}\left(d_{j}^{i}\right)_{m_{1}} m_{i} !}\right\}
\end{aligned}
$$

where for absolute convergence it is sufficient that

$$
1+q+q_{k}-p-p_{k} \geq 0 ; \quad k=1,2, \cdots, n
$$

The function in (3) is a special case of the $H$-functions of several variables which were defined in $[3,6]$. The fundamental theorem leads to the following result which involves the $G$-function of Meijer [8]. Let

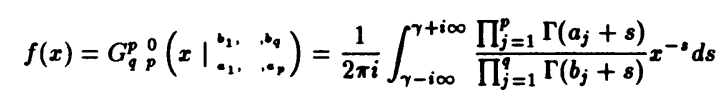

where $\operatorname{Re} a_{j}>0, j=1,2, \cdots, p$; and either $p=q$ and $\operatorname{Re} \sum_{j=1}^{p} a_{j}<\operatorname{Re} \sum_{j=1}^{q} b_{j}$ or $q<p$. It is known [5] that

$$
f^{*}(s)=\frac{\prod_{j=1}^{p} \Gamma\left(a_{j}+s\right)}{\prod_{j=1}^{q} \Gamma\left(b_{j}+s\right)}, \quad \operatorname{Re} s>0
$$

Therefore (2) becomes

$$
\begin{aligned}
& \int_{0}^{\infty} \cdots \int_{0}^{\infty} G_{q p}^{p 0}\left(\left.\max \left\{x_{1}, \cdots, x_{n}\right\}\right|_{\bullet_{1}, \cdots, c_{p}} ^{b_{1}, \omega_{p}}\right) \prod_{i=1}^{n} x_{i}^{s_{1}-1} d x_{1} \cdots d x_{n}= \\
& =\frac{\prod_{j=1}^{p} \Gamma\left(a_{j}+s_{1}+\cdots+s_{n}\right)}{\prod_{j=1}^{q} \operatorname{Gamma}\left(b_{j}+s_{1}+\cdots+s_{n}\right)} \frac{\left(s_{1}+\cdots+s_{n}\right)}{s_{1} \cdots s_{n}}, \quad \operatorname{Res}_{1}, \cdots, \operatorname{Re} s_{n}>0 .
\end{aligned}
$$

The representation for the generalized Lauricella function can now be obtained as follows. Let $p_{i} \leq q_{i}, i=$ $1,2, \cdots, n$, and either $p=q$ and $\operatorname{Re} \sum_{j=1}^{p} a_{j}<\operatorname{Re} \sum_{j-1}^{q} b_{j}$ or $p>q$. Then

$$
\begin{aligned}
& F_{q . q_{1} ; \cdot ; q_{n}}^{p . p_{n}}\left(\begin{array}{l}
a_{p} ; c_{p_{1}}^{1} ; \cdots ; c_{p_{n}}^{n} ; x_{1}, \cdots, x_{n} \\
b_{q} ; d_{q_{1}}^{1} ; \cdots ; d_{q_{n}}^{n} ;
\end{array}\right) \\
& =\frac{1}{n} \sum_{m_{1}, \cdots, m_{n}=0}^{\infty} \frac{\prod_{j=1}^{p}\left(a_{j}\right)_{m_{1}++m_{n}}(n)_{m_{1}+\cdots+m_{n}}\left(n+m_{1}+\cdots+m_{n}\right)}{\prod_{j=1}^{q}(b,)_{m_{1}}++m_{n}(n+1)_{m_{1}+}+m_{n}\left(m_{1}+1\right) \cdots\left(m_{n}+1\right)} . \\
& \cdot \prod_{i=1}^{n}\left\{\frac{\prod_{j=1}^{p_{1}}\left(c_{j}^{i}\right)_{m_{i}}(2)_{m_{1}} x_{i}^{m_{i}}}{\prod_{j=1}^{q_{i}}\left(d_{j}^{i}\right)_{m_{1}}(1)_{m_{1}} m_{i} !}\right\} \\
& =\frac{1}{n} \frac{\Gamma\left(b_{1}\right) \cdots \Gamma\left(b_{q}\right)}{\Gamma\left(a_{1}\right) \cdots \Gamma\left(a_{p}\right)} \int_{0}^{\infty} \cdots \int_{0}^{\infty} G_{q+1, p+1}^{p+1,0}\left(\left.\max \left\{t_{1}, \cdots, t_{n}\right\}\right|_{c_{1}-\infty, \cdots,-\infty, 0} ^{b_{1}-n, \cdots, b_{q}-n, 1}\right) . \\
& \cdot \prod_{i=1}^{n}\left\{\sum_{m_{1}=0}^{\infty} \frac{\prod_{j=1}^{p_{i}}\left(c_{j}^{2}\right)_{m_{1}}(2)_{m_{1}}\left(x_{i} t_{i}\right)^{m_{i}}}{\prod_{j=1}^{q_{i}}\left(d_{j}^{2}\right)_{m_{i}}(1)_{m_{1}} m_{i} !}\right\} d t_{1} \cdots d t_{n} .
\end{aligned}
$$


Thus we have proved

$$
\begin{aligned}
& F_{q}^{p q_{1},, p_{n}}\left(\begin{array}{l}
a_{p} ; c_{p_{1}}^{1} ; \cdots ; c_{p_{n}}^{n} ; x_{1}, \cdots, x_{n} \\
b_{q} ; d_{q_{1}}^{1} ; \cdots ; d_{q_{n}}^{n} ;
\end{array}\right)= \\
& =\frac{1}{n} \frac{\Gamma\left(b_{1}\right) \cdots \Gamma\left(b_{q}\right)}{\Gamma\left(a_{1}\right) \cdots \Gamma\left(a_{p}\right)} \cdot \subset n t_{0}^{\infty} \cdots \int_{0}^{\infty} G_{q+1, p+1}^{p+1,0}\left(\left.\max \left\{t_{1}, \cdots, t_{n}\right\}\right|_{c_{1}-n,} ^{b_{1}-n,}, \ldots,-\infty,-\infty, 0\right) . \\
& \cdot \prod_{i=1}^{n} p_{1+1} F_{q_{1}+1}\left(\begin{array}{c}
c_{1}^{i}, \cdots, c_{p_{1}}^{i}, 2 ; \\
d_{1}^{i}, \cdots, d_{q,}^{i}, 1 ;
\end{array} x_{i} t_{i}\right) d t_{1} \cdots d t_{n} .
\end{aligned}
$$

Formula (5) is valid when $\operatorname{Re}_{j}>n ; j=1,2, \cdots, p ; p_{i} \leq q_{i}, i=1,2, \cdots, n ; p \geq q, 1+q+q_{i}-p-p_{i} \geq 0 ; i=1,2, \cdots, n$. (The restriction $\operatorname{Re} \sum_{j=1}^{q} b_{j}+1>\operatorname{Re} \sum_{j=1}^{p} a_{j}$ is needed when $p=q$.)

\section{INTEGRAL REPRESENTATIONS OF LAURICELLA HYPERGEOMETRIC FUNCTIONS.}

Derivations similar to those in the previous section lead to representations for 3 of the Lauricella functions. (Since in (5) $p \geq q$ we do not get $F_{B}^{(n)}$.) We introduce the operator

$$
\mathcal{D}_{i}=\left(\frac{a-1}{n}+x_{i} \frac{d}{d\left(x_{i}\right)}\right)
$$

(a) Using the formula (2) we get

$$
\begin{gathered}
\int_{0}^{\infty} \cdots \int_{0}^{\infty} \exp \left(-\max \left\{x_{1}, \cdots, x_{n}\right\}\right) \prod_{i=1}^{n} x_{i}^{\frac{-1-1}{n}+m_{1}-1} d x_{1} \cdots d x_{n}= \\
=\frac{\Gamma\left(a+m_{1}+\cdots+m_{n}\right)}{\left(\frac{a-1}{n}+m_{1}\right) \cdots\left(\frac{a-1}{n}+m_{n}\right)}, \quad \text { Rea }>1 .
\end{gathered}
$$

Consequently, for the Lauricella function $F_{A}^{(n)}$ we have

$$
\begin{aligned}
& F_{A}^{(n)}\left(a ; b_{1}, \cdots, b_{n} ; c_{1}, \cdots, c_{n} ; x_{1}, \cdots, x_{n}\right)= \\
& =\sum_{m_{1}, m_{n}=0}^{\infty} \frac{(a)_{m_{1}}++m_{n}\left(b_{1}\right)_{m_{1}} \cdots\left(b_{n}\right)_{m_{n}}}{\left(c_{1}\right)_{m_{1}} \cdots\left(c_{n}\right)_{m_{n}} m_{1} ! \cdots m_{n} !} x_{1}^{m_{1}} \cdots x_{n}^{m_{n}}= \\
& =\sum_{m_{1},}^{\infty} \frac{1}{\Gamma\left(m_{n}=0\right.} \int_{0}^{\infty} \cdots \int_{0}^{\infty} \prod_{i=1}^{n} t_{i}^{\frac{-1-1}{n}+m_{1}-1} \exp \left(-\max \left\{t_{1}, \cdots, t_{n}\right\}\right) \\
& \cdot \prod_{i=1}^{n} \frac{\left(\frac{a-1}{n}+m_{i}\right)\left(b_{i}\right)_{m_{1}} x_{i}^{m_{1}}}{\left(c_{i}\right)_{m_{1}} m_{i} !} d t_{1} \cdots d t_{n}= \\
& =\frac{1}{\Gamma(a)} \int_{0}^{\infty} \cdots \int_{0}^{\infty}\left(t_{1} \cdots t_{n}\right)^{\frac{--1}{n}-1} \exp \left(-\max \left\{t_{1}, \cdots, t_{n}\right\}\right) \\
& \cdot \prod_{i=1}^{n}\left\{\sum_{m_{1}=0}^{\infty}\left(\frac{a-1}{n}+m_{i}\right) \frac{\left(b_{i}\right)_{m_{i}}\left(t_{i} x_{i}\right)^{m_{1}}}{\left(c_{\imath}\right)_{m_{1}} m_{i} !}\right\} d t_{1} \cdots d t_{n} \text {, }
\end{aligned}
$$

Consequently

$$
\begin{gathered}
F_{A}^{(n)}\left(a ; b_{1}, \cdots, b_{n} ; c_{1}, \cdots, c_{n} ; x_{1}, \cdots, x_{n}\right)= \\
=\frac{1}{\Gamma(a)} \prod_{i=1}^{n} \mathcal{D}_{i} \int_{0}^{\infty} \cdots \int_{0}^{\infty} \prod_{i=1}^{n} t_{i}^{\frac{a-1}{n}-1} \exp \left(-\max \left\{t_{1}, \cdots, t_{n}\right\}\right) \cdot \prod_{i=1}^{n}{ }_{1} F_{1}\left(b_{i}, c_{i} ; x_{i} t_{i}\right) d t_{1} \cdots d t_{n}, \\
\operatorname{Rea}>1 .
\end{gathered}
$$

(b) From (2) we have

$$
\begin{gathered}
\int_{0}^{1} \cdots \int_{0}^{1}\left(1-\max \left\{x_{1}, \cdots, x_{n}\right\}\right)^{b-a} \prod_{i=1}^{n} x_{i}^{\frac{a-1}{n}+m_{1}-1} d x_{1} \cdots d x_{n}= \\
=\frac{\Gamma\left(a+m_{1}+\cdots+m_{n}\right) \Gamma(b-a+1)}{\Gamma\left(b+m_{1}+\cdots+m_{n}\right)\left(\frac{a-1}{n}+m_{1}\right) \cdots\left(\frac{a-1}{n}+m_{n}\right)}, \quad 1+\operatorname{Re} b>\operatorname{Re} a>1 .
\end{gathered}
$$


Therefore for the Lauricella function $F_{D}^{(n)}$ we get

$$
\begin{aligned}
& F_{D}^{(n)}\left(a ; b_{1}, \cdots, b_{n} ; c ; x_{1}, \cdots, x_{n}\right)= \\
& =\sum_{m_{1}, m_{n}=0}^{\infty} \frac{(a)_{m_{1}+}+m_{n}\left(b_{1}\right)_{m_{1}}\left(b_{n}\right)_{m_{n}} x_{1}^{m_{1}} \cdots x_{n}^{m_{n}}}{(c)_{m_{1}}++m_{n} m_{1} ! \cdots m_{n} !}= \\
& =\frac{\Gamma(c)}{\Gamma(a) \Gamma(c-a+1)} \int_{0}^{1} \cdots \int_{0}^{1} \prod_{t=1}^{n} t_{i}^{\frac{--1}{n}-1}\left(1-\max \left\{t_{1}, \cdots, t_{n}\right\}\right)^{c-a} \\
& \prod_{i=1}^{n} \sum_{m_{1}=0}^{\infty}\left(\frac{a-1}{n}+m_{2}\right) \cdot \frac{\left(b_{\imath}\right)_{m_{1}}\left(x_{2} t_{z}\right)^{m}}{m_{2} !} d t_{1} \cdots d t_{n} .
\end{aligned}
$$

Hence

$$
\begin{gathered}
F_{D}^{(n)}\left(a ; b_{1}, \cdots, b_{n} ; c ; x_{1}, \cdots, x_{n}\right) \\
=\frac{\Gamma(c)}{\Gamma(a) \Gamma(c-a+1)} \prod_{t=1}^{n} \mathcal{D}_{2} \int_{0}^{1} \cdots \int_{0}^{1} \prod_{i=1}^{n} t_{t}^{\frac{--1}{n}-1}\left(1-\max \left\{t_{1}, \cdots, t_{n}\right\}\right)^{c-a} \prod_{i=1}^{n}\left(1-x_{2} t_{2}\right)^{-b \cdot} d t_{1} \cdots d t_{n},
\end{gathered}
$$

$$
1+\operatorname{Rec}>\operatorname{Re} a>1
$$

(c) Using the formula (2) again, we have

$$
\begin{gathered}
\int_{0}^{\infty} \cdots \int_{0}^{\infty}\left(\max \left\{x_{1}, \cdots, x_{n}\right\}\right)^{\frac{b-a+1}{2}} K_{b-a+1}\left(2\left(\max \left\{t_{1} \cdots t_{n}\right\}\right)^{\frac{1}{2}}\right) \prod_{i=1}^{n}\left(x_{i}\right)^{\frac{--1}{n}+m_{1}-1} d x_{1} \cdots d x_{n}= \\
=\frac{1}{2} \frac{\Gamma\left(a+m_{1}+\cdots+m_{n}\right) \Gamma\left(b+m_{1}+\cdots+m_{n}\right)}{\left(\frac{a-1}{n}+m_{1}\right) \cdots\left(\frac{a-1}{n}+m_{n}\right)}, \quad \text { Rea }>1, \text { Reb }>-0 .
\end{gathered}
$$

Consequently, for the Lauricella hypergeometric function $F_{C}^{(n)}$, by a similar development we have the result

$$
\begin{gathered}
F_{C}^{(n)}\left(a ; b ; c_{1}, \cdots, c_{n} ; x_{1}, \cdots, x_{n}\right)= \\
=\frac{2}{\Gamma(a) \Gamma(b)} \prod_{i=1}^{n} \mathcal{D}_{i} \int_{0}^{\infty} \cdots \int_{0}^{\infty} \prod_{i=1}^{n} t_{i}^{\frac{-1-1}{n}-1}\left(\max \left\{t_{1}, \cdots, t_{n}\right\}\right)^{\frac{b-a+1}{2}} K_{b-a+1}\left(2\left(\max \left\{t_{1} \cdots t_{n}\right\}\right)^{\frac{1}{2}}\right) \\
\prod_{i=1}^{n}{ }_{0} F_{1}\left(c_{i} ; x_{i} t_{i}\right) d t_{1} \cdots d t_{n}, \\
\text { Rea }>1, \quad \text { Reb }>-1 .
\end{gathered}
$$

For Appell functions these read

$$
\begin{gathered}
F_{1}\left(a ; b_{1}, b_{2} ; c ; x_{1}, x_{2}\right)=\frac{\Gamma(c)}{\Gamma(a) \Gamma(c-a+1)} \\
\mathcal{D}_{1} \mathcal{D}_{2} \int_{0}^{1} \int_{0}^{1}\left(1-\max \left\{t_{1}, t_{2}\right\}\right)^{c-a}\left(t_{1} t_{2}\right)^{(a-3) / 2}\left\{\left(1-x_{1} t_{1}\right)^{-b_{1}}\right\}\left\{\left(1-x_{2} t_{2}\right)^{-b_{2}}\right\} d t_{1} d t_{2}, \\
1+\operatorname{Re} c>\operatorname{Re} a>1 ; \\
=\frac{1}{\Gamma(a)} \mathcal{D}_{1} \mathcal{D}_{2} \int_{0}^{\infty} \int_{0}^{\infty}\left(t_{1} t_{2}\right)^{(a-3) / 2} \exp \left(-\max \left\{t_{1}, t_{2}\right\}\right) \\
\left\{F_{1}\left(b_{1} ; c_{1} ; x_{1} t_{1}\right)\right\}\left\{F_{1} F_{1}\left(b_{2} ; c_{2} ; x_{2} t_{2}\right)\right\} d t_{1} d t_{2}, \\
\operatorname{Rea}>1 ;
\end{gathered}
$$




$$
\begin{gathered}
F_{4}\left(a ; b ; c_{1}, c_{2} ; x_{1}, x_{2}\right)= \\
=\frac{2 \Gamma\left(c_{1}\right) \Gamma\left(c_{2}\right)}{\Gamma(a) \Gamma(b)} \mathcal{D}_{1} \mathcal{D}_{2} \int_{0}^{\infty} \int_{0}^{\infty}\left(\max \left\{t_{1}, t_{2}\right\}\right)^{(b-a+1) / 2}\left(t_{1} t_{2}\right)^{(a-3) / 2} K_{b-a+1}\left(2\left(\max \left\{t_{1}, t_{2}\right\}\right)^{1 / 2}\right) \\
\cdot\left\{\left(x_{1} t_{1}\right)^{\left(1-c_{1}\right) / 2} I_{c_{1}-1}\left(2\left(x_{1} t_{1}\right)^{1 / 2}\right)\right\}\left\{\left(x_{2} t_{2}\right)^{\left(1-c_{2}\right) / 2} I_{c_{2}-1}\left(2\left(x_{2} t_{2}\right)^{1 / 2}\right)\right\} d t_{1} d t_{2}, \\
\text { Rea }>1, \text { Reb }>0 .
\end{gathered}
$$

\section{REFERENCES}

1. P. APPEll AND J. Kampé De FÉRIET, Fonctions hypergéométriques et hypersphériques, Polynomes d'Hermite, Gauthier-Villars, Paris, 1926.

2. Yu. A. Brychkov, H.J. Glaeske, A.P. Prudnikov, and VU Kim TUan, Multidımensional Integral Transforms, Geest \& Portig K.G., Leipzig and D. Reidel Publishing Company, Amsterdam, 1990.

3. R.G. Buschman, The H-functions of $N$ variables, Ranchi Univ. Math. J. 10 (1979), 81-88.

4. V.M. ĊERNov, Some questions of the operational calculus concerning the two-sided, two-dimensional LaplaceCarson transform, Izv. Vysš. Učebn. Zaved. Mat. 2 (33) (1963), 140-151.

5. O.I. Marichev, Handbook of Integral Transforms of Higher Transcendental Functions, Theory and Algorithmic Tables, Ellis Horwood, Chichester, 1982.

6. O.I. MARIChev and VU KIM TUAN, The problems of definitions and symbols of $G$ - and $H$-functions of several variables, Rev. Tecn. Fac. Ing. Univ. Zulia, Special Edition 6 (1983), 144-151.

7. H.M. SRivastava and M.C. Daoust, Certain generalized Neumann expansions associated with the Kampé de Fériet function, Nederl. Akad. Wetensch. Proc. A72 (1969), 449-457.

8. H.M. SRivastava, K.C. Gupta, AND S.P. Goyal, The H-Functions of One and Two Variables with Applicatıons, South Asian Publishers Ltd., New Delhi, 1982.

9. D. Voelker Und G. Doetsch, Die Zweidimensionale Laplace Thansformation, Verlag Birkhäuser, Basel, 1950. 


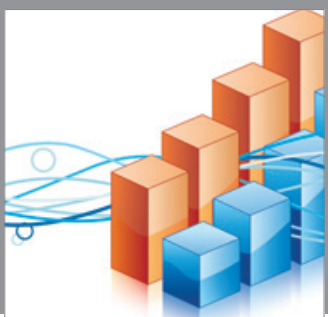

Advances in

Operations Research

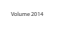

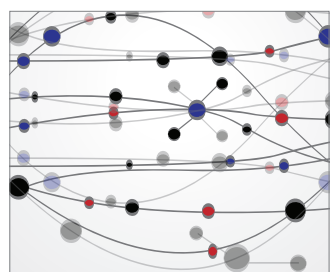

\section{The Scientific} World Journal
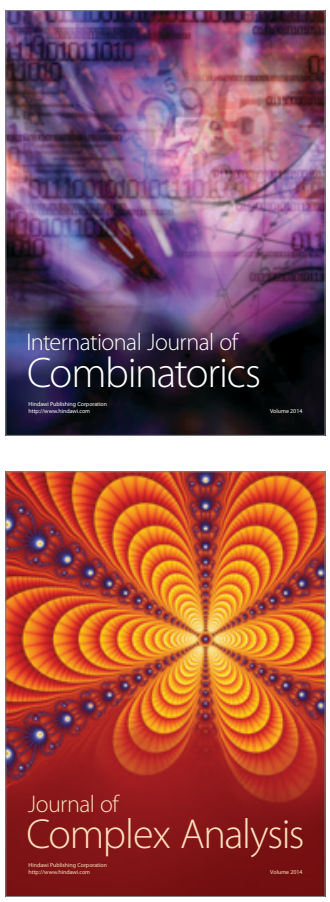

International Journal of

Mathematics and

Mathematical

Sciences
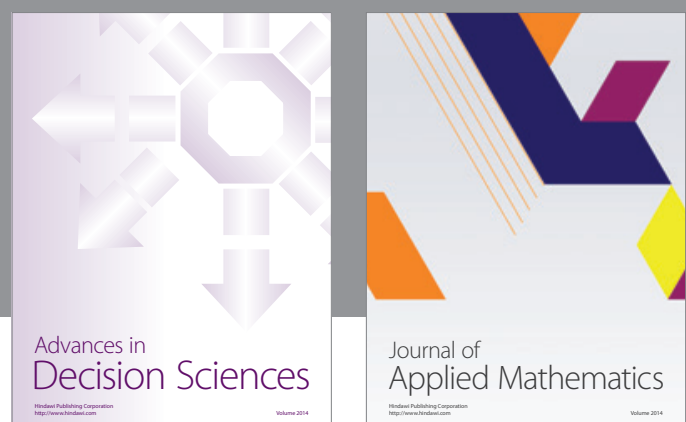

Journal of

Applied Mathematics
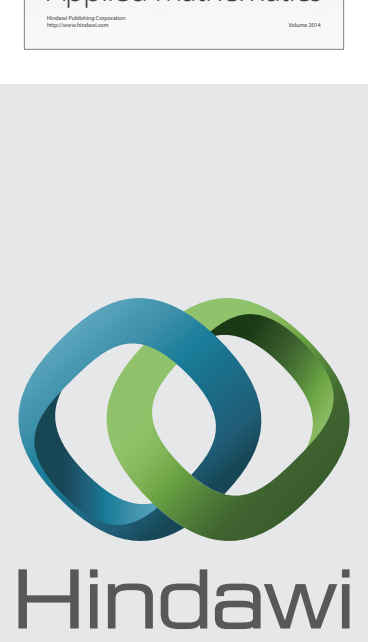

Submit your manuscripts at http://www.hindawi.com
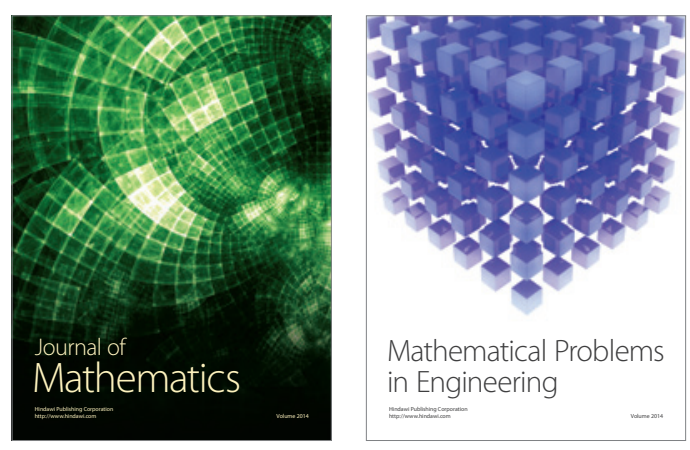

Mathematical Problems in Engineering
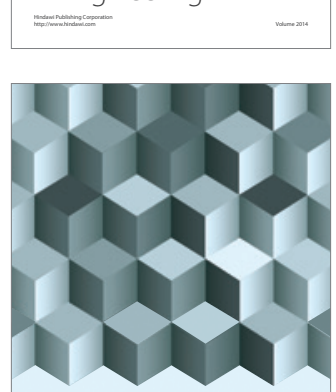

Journal of

Function Spaces
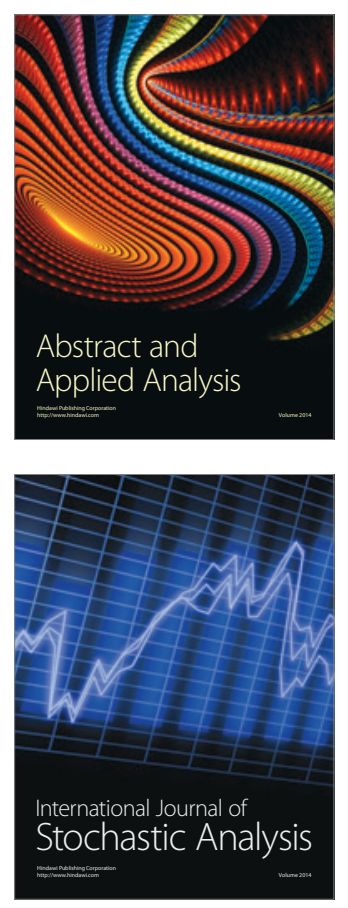

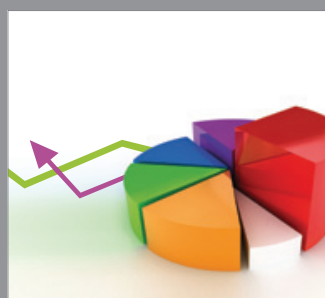

ournal of

Probability and Statistics

Promensencen
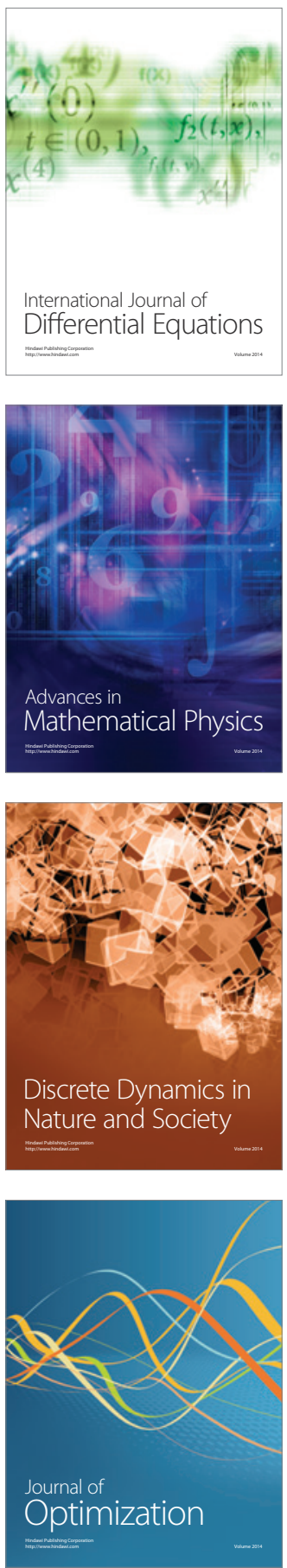